2011

\title{
Investigation of diarrhoea in a traveller just returned from India
}

Nicholas Arnold Zwar

University of New South Wales, nzwar@uow.edu.au

Adrienne Torda

Prince of Wales Hospital

\section{Publication Details}

Zwar, N. A. \& Torda, A. (2011). Investigation of diarrhoea in a traveller just returned from India. BMJ: British Medical Journal, 342 342-1-342-5.

Research Online is the open access institutional repository for the University of Wollongong. For further information contact the UOW Library: research-pubs@uow.edu.au 


\title{
Investigation of diarrhoea in a traveller just returned from India
}

\begin{abstract}
The causes of diarrhoea in people returning from travelling can be infective and non-infective, and the duration of symptoms is an important factor in identifying the cause. The article outlines the appropriate investigations
\end{abstract}

Keywords

india, traveller, just, returned, investigation, diarrhoea

Disciplines

Medicine and Health Sciences | Social and Behavioral Sciences

Publication Details

Zwar, N. A. \& Torda, A. (2011). Investigation of diarrhoea in a traveller just returned from India. BMJ: British Medical Journal, 342 342-1-342-5. 


\title{
Investigation of diarrhoea in a traveller just returned from India
}

The causes of diarrhoea in people returning from travelling can be infective and non-infective, and the duration of symptoms is an important factor in identifying the cause. The article outlines the appropriate investigations

\author{
Nicholas A Zwar professor of general practice ${ }^{1}$, Adrienne Torda senior staff specialist ${ }^{2}$
}

${ }^{1}$ School of Public Health and Community Medicine, University of New South Wales, Sydney, NSW 2052, Australia; ${ }^{2}$ Prince of Wales Hospital, Barker Street, Randwick, NSW 2031, Australia

This series of occasional articles provides an update on the best use of key diagnostic tests in the initial investigation of common or important clinical presentations. The series advisers are Steve Atkin, professor, head of department of academic endocrinology, diabetes, and metabolism, Hull York Medical School; and Eric Kilpatrick, honorary professor, department of clinical biochemistry, Hull Royal Infirmary, Hull York Medical School. To suggest a topic for this series, please email us at practice@bmj.com.

A previously well 21 year old student who has just returned from a three week trip to India presents with diarrhoea. In her final week there she did volunteer work in a rural village. Her symptoms started five days ago, with three to four unformed motions a day, fever, and cramps. Her fever has resolved but loose motions continue. A physical examination is normal except for active bowel sounds.

\section{What is the next investigation?}

Definitions differ slightly, but classic travellers' diarrhoea is usually defined as three or more unformed stools within 24 hours passed by a traveller and accompanied by other symptoms - most often cramps, nausea, fever, blood with the stools, vomiting, and faecal urgency. More than 15 million travellers are estimated to experience diarrhoea each year ${ }^{1}$; developing countries in particular are high risk destinations, with rates of diarrhoea between $20 \%$ and $90 \%$ for each two week stay, usually in the first week of travel. Travellers' diarrhoea often disrupts planned activities. ${ }^{1}$

Though travellers are often told to "boil it, cook it, peel it, or forget it," the effectiveness of personal hygiene and dietary precautions in preventing travellers' diarrhoea has been questioned, with a review reporting that seven of eight studies found no evidence that these personal hygiene precautions prevented or reduced the risk of developing travellers' diarrhoea. ${ }^{2}$ The single prospective study on the ability of travellers to follow strict hygiene advice found that by three days into their trip $98 \%$ of Swiss travellers who responded had been unable to follow such advice. ${ }^{3}$

The causes of diarrhoea in travellers can be both infective and non-infective. The duration of symptoms is an important factor in thinking about the likely cause. About $50-75 \%$ of acute cases of travellers' diarrhoea (duration less than two weeks) are caused by bacteria. ${ }^{4}$ The most common bacterial cause is enterotoxic Escherichia coli. Other common bacterial causes include campylobacter, salmonella, and shigella. Viruses, most commonly rotaviruses and noroviruses, account for $10-20 \%$ of cases, and parasitic agents such a giardia, cryptosporidium, and cyclospora account for up to $5 \%$ of cases. ${ }^{4}$ Vibrio cholerae is an uncommon cause of acute travellers' diarrhoea but is worth considering if the patient has travelled in the area of an outbreak. Even with careful laboratory investigation, however, no pathogen is detected in a substantial proportion of cases. Diarrhoea can also be a presenting feature of sepsis from a range of causes including pneumonia and urinary tract infection, as well as other travel related infections including typhoid fever (though this more often causes constipation). If the traveller is febrile and has been to a malarial area, consider the risk of malaria. ${ }^{5}$

When diarrhoea has been present for more than two weeks, the spectrum of possible causes is wider and non infective causes become increasingly important. Taking a good travel and clinical history is paramount. Features of the stool may be of help. Blood and mucus is characteristic of dysentery, which can be caused by a range of pathogens including shigella and amoeba. Bulky stools suggest giardia or malabsorption. However, stool microscopy and culture is the key investigation.

The triple faeces test (collection of faeces specimens on three consecutive days) has been shown to increase substantially the 


\section{Learning points}

The likely cause of travellers' diarrhoea depends on the duration of symptoms

Bacteria, in particular enterotoxic Escherichia coli, are the most common pathogens found in travellers with acute symptoms (less than two weeks' duration)

Stool microscopy for ova, cysts, and parasites and culture (ideally three specimens) form the key investigation in a patient who has just returned from travelling and has diarrhoea, although a causative agent is often not found

If symptoms persist, investigate for less common pathogens, such as parasites, and for non-infective causes

diagnostic yield of parasites ${ }^{67}$ but is often not practical. The stool should be examined for ova cysts and parasites and be cultured for bacteria, with viral antigen detection tests conducted. Stool specimens are not easy to collect in general practice. Patients can collect a specimen by putting plastic film over the toilet bowel under the seat, then scooping some stool into the sample jar. The stool sample needs to get to the laboratory immediately to have the best chance of pathogens being identified. The specimen should be refrigerated only if a delay of more than six hours is likely. Most laboratories have a "stool collection kit," which includes patient instructions and one plain plastic container and one container with fixative (for parasite identification). Other tests (table 1) can be done on the sample in the plain container. Communication with the laboratory may be needed to ensure appropriate samples are collected, and it is important to provide the pathologist with relevant clinical information.

Systemic infections may present with diarrhoea and may need to be excluded in a traveller returning from a foreign place. Other investigations will be influenced by the history and clinical circumstances (table 2).

\section{Outcome}

Rehydration is the main aspect of treatment. Antimotility agents are often self administered but are best avoided if there is fever and bloody diarrhoea as they may prolong the illness. ${ }^{4}$ Antimicrobials are often of limited benefit in reducing the mean number of stools a day but may shorten the duration of the illness in the acute phase. ${ }^{9}{ }^{10}$ Limiting use to cases in which symptoms are moderate (more than two stools per 24 hours and distressing symptoms) to severe (fever or bloody stools, more than six stools in 24 hours) is recommended.$^{11}$ Resistance to antibiotics has been increasing and is common to doxycyline and co-trimoxazole (a mixture of trimethoprim and sulfamethoxazole). ${ }^{9}$ Quinolones are most commonly used for empirical treatment in non-pregnant adults, but azithromycin may be preferred for treating travellers to South East Asia and India owing to rising rates of resistance to quinolones in campylobacter species. ${ }^{11}$

In the case above, the stool microscopy and culture did not identify any causative agent. Her symptoms improved over several days, but a month later she described irregular bowel habit and abdominal bloating. Once symptoms become chronic (lasting more than two weeks) or relapsing, non-infective problems need to be considered. Table 3 gives a list of the most common causes.

Tests of the stool for reducing substances were normal. Further stool examinations including antigen tests for ova, cysts, and parasites were negative. Serological tests on blood for antibodies against Entamoeba histolytica, Strongyloides stercoralis, and schistosoma were negative. A coeliac screen with anti-gliadin antibodies and transglutaminase antibody $\operatorname{Ig} \mathrm{A}$ was negative. A diet low in lactose made no noticeable difference to symptoms, and the likely cause of the continued symptoms was thought to be irritable bowel syndrome.

In a prospective cohort study of 97 students travelling to Mexico, $10 \%$ of those who had travellers' diarrhoea subsequently developed symptoms of irritable bowel syndrome. ${ }^{12}$ Other research has found that 12 months after an episode of acute gastroenteritis (not necessarily associated with travel), between $4 \%$ and $31 \%$ of people have post-infectious irritable bowel syndrome. ${ }^{13}$ General advice includes reduction of caffeine intake and increased dietary fibre. In this patient the symptoms resolved after 12 months.

Contributors: NAZ did the initial literature searches and wrote the first draft. AT provided expert comment and advice from the perspective of an infectious disease specialist. Both authors critically revised subsequent drafts. NAZ is the guarantor.

Competing interests: All authors have completed the Unified Competing Interest form at www.icmje.org/coi_disclosure.pdf (available on request from the corresponding author) and declare: no support from any organisation for the submitted work; no financial relationships with any organisations that might have an interest in the submitted work in the previous three years; no other relationships or activities that could appear to have influenced the submitted work.

Provenance and peer review: Commissioned; externally peer reviewed. Patient consent not required (patient anonymised, dead, or hypothetical).

Steffen R. Epidemiology of travelers'diarrhea. CID 2005;41:S536-40.

2 Shlim D. Looking for evidence that personal hygiene precautions prevent travellers' diarrhea. CID 2005;41:S531-35.

3 Kozicki M, Steffen R, Schar M. "Boil it, cook it, peel it or forget it": does this rule prevent travellers' diarrhoea? Int J Epidemiology 1985;14:169-72.

4 Ryan ET, Wilson ME, Kain KC. Current concepts: illness after international travel. N Engl J Med 2002;347:505-16

5 Nield VF, Ford L, Hill DR, eds. Health information for overseas travel. National Travel Health Network and Centre, 2010. www.nathnac.org/yellow_book/YBmainpage.htm

6 Rohner P, Pittet D, Pepey B, Nije-Kinge T, Auckenthaler R. Etiological agents of infectious diarrhea: implications for requests for microbiological culture. J Clin Microbiol 1997;35:1427-32.

7 Van Gool T, Weijts R, Lommerse E, Mank TG. Triple Faeces Test: an effective tool for detection of intestinal parasites in routine clinical practice. Eur J Clin Microbiol Infect Dis 2003 May;22(5):284-90.

8 Patel U, Gandhi G, Friedman S, Niranjan S. Thrombocytopenia in malaria. J Nat Med Assoc 2004;96:1212-1214.

9 Ericsson CD. Travelers' diarrhea: epidemiology, prevention and self -treatment. Infect Dis Clin North Am 1998;12:285-303.

10 De Bruyn G, Hahn S, Borwick A. Antibiotic treatment for travellers' diarrhoea. Cochrane Database Syst Rev 2000;3:CD002242.

11 Ostrosky-Zeichner L, Ericsson CD, Travelers' diarrhea. In: Zuckerman JN, editor. Principles and practice of travel medicine. Chichester: John Wiley and Sons; 2001. p. 154

12 Okhuysen PC, Jiang ZD, Carlin L, et al. Post-diarrhea chronic intestinal symptoms and irritable bowel syndrome in North American travellers to Mexico. Am J Gastroenterol 2004;99:1774-78.

13 Connor B. Sequelae of travelers'diarrhea: focus on postinfectious irritable bowel syndrome CID 2005;41:S577-86. 


\section{Tables}

Table 1/ Stool investigations in travellers' diarrhoea

\section{Investigation}

Stool microscopy for ova, cysts, and parasites

Stool culture and viral antigen testing

Test stool for Clostridium difficile toxin

Test stool for disaccharides (reducing substances)

\section{Comments}

Sensitivity varies depending on the parasite but generally exceeds $80 \%$

Sensitivity is increased by examining several samples ${ }^{7}$

Bacterial or viral cause can been identified in $50-70 \%$ of travellers who have diarrhoea lasting $<2$ weeks If antibiotics have been used in the past month

If the patient has clinical features of malabsorption or persisting diarrhoea. If so, a test of stool acidity can be used to identify lactase or sucrase deficiency 
Table 2| Basic investigations other than stool microscopy and culture

\section{Investigation}

Full blood count, blood film, and differential

Serum electrolyte concentrations and renal function tests

Liver function tests

Blood films for malaria parasites or immunochromatography test

\section{Comments}

Useful in investigating for systemic infections and may prompt further specific investigations. For example, white cell count may be low in overwhelming sepsis and malaria. Eosinophilia suggests parasitic infection. Thrombocytopenia is present in $40-85 \%$ of patients with malaria ${ }^{8}$

Raised in systemic infections

Important if dehydration is suspected

Raised transaminase and bilirubin levels may indicate hepatitis, amoebiasis, or septicaemia

Diarrhoea is present in about $20 \%$ of people with malaria. Malaria is not excluded on a single film, and examination of three blood films is recommended if malaria is suspected 


\section{Table 3| Non-infective causes of diarrhoea}

\section{Non-infective causes}

\section{Characteristics}

Post-infective malabsorption Tends to get better over weeks, especially with elimination of exacerbating foods such as dairy products

Irritable bowel syndrome

Lactose intolerance

Tropical sprue

Patient will have a suggestive clinical history and usually chronic symptoms; the syndrome is often a diagnosis of exclusion

Symptoms are exacerbated by intake of dairy products; investigations may show disaccharides in stool and a positive hydrogen breath test

Patient will have a history consistent with this diagnosis, such as steatorrhoea, cramps, and weight loss; suggestive features on bowel biopsy

Inflammatory bowel disease Usually diagnosed on bowel biopsy \begin{tabular}{ll} 
Coeliac disease & $\begin{array}{l}\text { Patient will usually have history of symptoms predating travel and may have a family history. Arrange confirmatory blood tests } \\
\text { (anti-gliaden and anti-transglutaminase antibodies) and biopsy after gluten challenge }\end{array}$ \\
\hline
\end{tabular}

Malignancy More likely in older patients and those with ongoing symptoms and when diarrhoea is accompanied by rectal bleeding and weight loss

Hyperthyroidism Abnormalities on thyroid function tests (the patient may also have other symptoms of hyperthyroidism) 